\title{
A note on fixed point theory for cyclic $\varphi$-contractions
}

\section{Stojan Radenovic ${ }^{*}$}

\section{"Correspondence:}

fixedpoint50@gmail.com

Faculty of Mathematics and

Information Technology, Teacher

Education, Dong Thap University,

Cao Lanch, Dong Thap, Vietnam

\begin{abstract}
In this paper, we consider, discuss, improve, and complement recent fixed points results for mappings satisfying cyclical contractive conditions established by Pacurar and Rus (Nonlinear Anal. 72:1181-1187, 2010) and Chandok and Postolache (Fixed Point Theory Appl. 2013:28, 2013). By using a new lemma we get much shorter and nicer proofs of some results with the new concept of mappings.
\end{abstract}

MSC: $47 \mathrm{H} 10 ; 54 \mathrm{H} 25$

Keywords: cyclic $\varphi$-contraction; Picard operator; comparison function; (c)-comparison function; weakly Chatterjea-type contraction

\section{Introduction and preliminaries}

It is well known that the Banach contraction principle [1] is one of the fundamental results in nonlinear analysis and fixed point theory, in general. It has various applications in many branches of applied sciences. Also, there are several extensions and generalizations of this principle. For example, Kirk et al. [2] obtained an extension of the Banach principle for mappings satisfying cyclical contractive conditions. They also proved in [2] Edelstein's, Boyd-Wong, Geraghty and Caristy type theorem for new concept of mappings. For some other generalizations also see $([3,4]$ and $[5])$.

Following [2], in 2010 [6], Pacurar and Rus proved a fixed point theorem for cyclic $\varphi$-contractions. Also, very recent, following ideas from [2], Chandok and Postolache [7] proved a fixed point theorem for weakly Chatterjea-type cyclic contractions.

However, in the present paper, we show that all these results established in [6] and [7] are in the fact equivalent with well-known ordinary fixed point results in literature.

Let us start by recalling a definition from [2, 7], and [6].

Definition 1.1 [2] Let $X$ be a non-empty set, $p \in \mathbb{N}$, and $f: X \rightarrow X$ a map. Then we say that $\bigcup_{i=1}^{p} A_{i}$ (where $\emptyset \neq A_{i} \subseteq X$ for all $i \in\{1,2, \ldots, p\}$ ) is a cyclic representation of $X$ with respect to $f$ if and only if the following two conditions hold:

(I) $X=\bigcup_{i=1}^{p} A_{i}$;

(II) $f\left(A_{i}\right) \subseteq A_{i+1}$ for $1 \leq i \leq p-1$, and $f\left(A_{p}\right) \subseteq A_{1}$.

In 2003 [2] Kirk et al. proved the following result.

(c) 2015 Radenović. This article is distributed under the terms of the Creative Commons Attribution 4.0 International License (http://creativecommons.org/licenses/by/4.0/), which permits unrestricted use, distribution, and reproduction in any medium, provided you give appropriate credit to the original author(s) and the source, provide a link to the Creative Commons license, and indicate if changes were made. 
Theorem 1.2 [2] Let $\left\{A_{i}\right\}_{i=1}^{p}$ be non-empty closed subsets of a complete metric space, and suppose $F: \bigcup_{i=1}^{p} A_{i} \rightarrow \bigcup_{i=1}^{p} A_{i}$ satisfies the following conditions (where $A_{p+1}=A_{1}$ ):

(1) $F\left(A_{i}\right) \subset A_{i+1}$ for $1 \leq i \leq p$;

(2) $\exists k \in(0,1)$ such that $d(F(x), F(y)) \leq k d(x, y) \forall x \in A_{i}, y \in A_{i+1}$ for $1 \leq i \leq p$.

Then $F$ has a unique fixed point.

Remark 1.3 It is not hard to see that Theorem 1.2 holds if $k=0$. Also, it easily follows that Picard's sequence $\left\{F^{n} x\right\}$ converges to a unique fixed point of $F$ for all $x \in X$.

Definition 1.4 [6] A function $\varphi:[0, \infty) \rightarrow[0, \infty)$ is called a comparison function if it satisfies:

(i) $\varphi$ $\varphi$ is increasing, i.e., $t_{1} \leq t_{2}$ implies $\varphi\left(t_{1}\right) \leq \varphi\left(t_{2}\right)$, for $t_{1}, t_{2} \in[0, \infty)$;

(ii) $\varphi_{\varphi}\left(\varphi^{n}(t)\right)_{n \in \mathbb{N}}$ converges to 0 as $n \rightarrow \infty$, for all $t \in(0, \infty)$.

There is a rich literature referring to $\varphi$-contractions, i.e., a self-operator defined on a metric space, which satisfies

$$
d(f(x), f(y)) \leq \varphi(d(x, y))
$$

for any $x, y \in X$, where $\varphi$ is a comparison function.

Definition 1.5 [6] A function $\varphi:[0, \infty) \rightarrow[0, \infty)$ is called a (c)-comparison function if it satisfies:

(i) $\varphi$ is increasing, i.e., $t_{1} \leq t_{2}$ implies $\varphi\left(t_{1}\right) \leq \varphi\left(t_{2}\right)$, for $t_{1}, t_{2} \in[0, \infty)$;

(ii) $)_{\varphi}$ there exist $k_{0} \in \mathbb{N}, a \in(0,1)$ and a convergent series of nonnegative terms $\sum_{k=1}^{\infty} v_{k}$ such that

$$
\varphi^{k+1}(t) \leq a \varphi^{k}(t)+v_{k}
$$

for $k \geq k_{0}$ and any $t \in(0, \infty)$.

The following result is well known.

Lemma 1.6 [6] If $\varphi:[0, \infty) \rightarrow[0, \infty)$ is a (c)-comparison function, then the following hold:

(i) $\varphi$ is a comparison function;

(ii) $\varphi(t)<t$, for any $t \in(0, \infty)$;

(iii) $\varphi$ is continuous at 0 ;

(iv) the series $\sum_{k=0}^{\infty} \varphi^{k}(t)$ converges for any $t \in(0, \infty)$.

Let $P_{c l}(X)$ denote the collection of non-empty closed subsets of $X$. Then we have the following.

Definition 1.7 [6] Let $(X, d)$ be a metric space, $m$ a positive integer, $A_{1}, \ldots, A_{m} \in P_{c l}(X)$, $Y=\bigcup_{i=1}^{m} A_{i}$, and $f: Y \rightarrow Y$ an operator. If:

(i) $\bigcup_{i=1}^{m} A_{i}$ is a cyclic representation of $Y$ w.r.t.f; 
(ii) there exists a comparison function $\varphi:[0, \infty) \rightarrow[0, \infty)$ such that

$$
d(f(x), f(y)) \leq \varphi(d(x, y))
$$

for any $x \in A_{i}, y \in A_{i+1}$, where $A_{m+1}=A_{1}$, then $f$ is a cyclic $\varphi$-contraction.

The main result of [6] is the following.

Theorem 1.8 [6] Let $(X, d)$ be a complete metric space, $m$ a positive integer, $A_{1}, \ldots, A_{m} \in$ $P_{c l}(X), Y=\bigcup_{i=1}^{m} A_{i}, \varphi:[0, \infty) \rightarrow[0, \infty) a(c)$-comparison function and $f: Y \rightarrow Y$ an operator. Assume that:

(i) $\bigcup_{i=1}^{m} A_{i}$ is a cyclic representation of $Y$ with respect to $f$;

(ii) $f$ is a cyclic $\varphi$-contraction.

Then:

(1) $f$ has a unique fixed point $x^{*} \in \bigcap_{i=1}^{m} A_{i}$ and the Picard iteration $\left\{x_{n}\right\}_{n \geq 0}$ given by $x_{n}=f\left(x_{n-1}\right), n \geq 1$ converges to $x^{*}$ for any starting point $x_{0} \in Y$.

(2) The following estimates hold:

$$
\begin{aligned}
& d\left(x_{n}, x^{*}\right) \leq \sum_{n=1}^{\infty} \varphi^{n}\left(d\left(x_{0}, x_{1}\right)\right) \\
& d\left(x_{n}, x^{*}\right) \leq \sum_{n=1}^{\infty} \varphi\left(d\left(x_{n}, x_{n+1}\right)\right) .
\end{aligned}
$$

(3) For any $x \in Y$ :

$$
d\left(x, x^{*}\right) \leq \sum_{n=1}^{\infty} \varphi^{k}(d(x, f(x)))
$$

It is worth to notice that Theorem 1.8 is a cyclical-type extension of the following ordinary fixed point theorem.

Theorem 1.9 Let $(X, d)$ be a complete metric space, and $f: X \rightarrow X$ an operator. Assume that there exists a (c)-comparison function $\varphi:[0, \infty) \rightarrow[0, \infty)$ such that

$$
d(f(x), f(y)) \leq \varphi(d(x, y))
$$

for all $x, y \in X$.

Then:

(1) $f$ has a unique fixed point $x^{*} \in X$ and the Picard iteration $\left\{x_{n}\right\}_{n \geq 0}$ given by $x_{n}=f\left(x_{n-1}\right), n \geq 1$ converges to $x^{*}$ for any starting point $x_{0} \in X$.

(2) The following estimates hold:

$$
\begin{aligned}
& d\left(x_{n}, x^{*}\right) \leq \sum_{n=1}^{\infty} \varphi^{n}\left(d\left(x_{0}, x_{1}\right)\right) \\
& d\left(x_{n}, x^{*}\right) \leq \sum_{n=1}^{\infty} \varphi\left(d\left(x_{n}, x_{n+1}\right)\right) .
\end{aligned}
$$


(3) For any $x \in X$ :

$$
d\left(x, x^{*}\right) \leq \sum_{n=1}^{\infty} \varphi^{k}(d(x, f(x)))
$$

Definition 1.10 [7] Let $\Phi$ denote the set of all monotone increasing continuous functions $\mu:[0, \infty) \rightarrow[0, \infty)$, with $\mu(t)=0$, if and only if $t=0$, and let $\Psi$ denote the set of all lower semi-continuous functions $\psi:[0, \infty)^{2} \rightarrow[0, \infty)$, with $\psi\left(t_{1}, t_{2}\right)>0$, for $t_{1}, t_{2} \in(0, \infty)$ and $\psi(0,0)=0$.

Definition 1.11 [7] Let $(X, d)$ be a metric space, $m$ be a natural number, $A_{1}, A_{2}, \ldots, A_{m}$, be non-empty subsets of $X$ and $Y=\bigcup_{i=1}^{m} A_{i}$. An operator $T: Y \rightarrow Y$ is called a weakly Chatterjea-type cyclic contraction if:

(1) $\bigcup_{i=1}^{m} A_{i}$ is a cyclic representation of $Y$ with respect to $T$;

(2) $\mu(d(T x, T y)) \leq \mu\left(\frac{1}{2}(d(x, T y)+d(y, T x))\right)-\psi(d(x, T y), d(y, T x))$ for all $x \in A_{i}, y \in A_{i+1}$, $i=1,2, \ldots, m$, where $A_{m+1}=A_{1}, \mu \in \Phi$, and $\psi \in \Psi$.

In [7] the authors proved the following result.

Theorem 1.12 [7] Let $(X, d)$ be a complete metric space, $m \in \mathbb{N}, A_{1}, A_{2}, \ldots, A_{m}$ be nonempty closed subsets of $X$ and $Y=\bigcup_{i=1}^{m} A_{i}$. Suppose that $T$ is a weakly Chatterjea-type cyclic contraction. Then Thas a fixed point $z \in \bigcap_{i=1}^{m} A_{i}$.

It is obvious that Theorem 1.12 is a cyclical-type extension of the following ordinary fixed point theorem.

Theorem 1.13 Let $(X, d)$ be a complete metric space, $T: X \rightarrow X$ be (so-called Chatterjeatype contraction) such that

$$
\mu(d(T x, T y)) \leq \mu\left(\frac{1}{2}(d(x, T y)+d(y, T x))\right)-\psi(d(x, T y), d(y, T x))
$$

for all $x, y \in X, \mu \in \Phi$ and $\psi \in \Psi$. Then $T$ has a unique fixed point.

\section{Main results}

Here we will prove and use the following (new, useful, and very significant) result in proofs of cyclic-type results (see also the proof of [3], Theorem 2.1 as well as [8], Lemma 2.1).

Lemma 2.1 Let $(X, d)$ be a metric space, $f: X \rightarrow X$ be a mapping and let $X=\bigcup_{i=1}^{p} A_{i}$ be a cyclic representation of $X$ w.r.t.f. Assume that

$$
\lim _{n \rightarrow \infty} d\left(x_{n}, x_{n+1}\right)=0
$$

where $x_{n+1}=f x_{n}, x_{1} \in A_{1}$. If $\left\{x_{n}\right\}$ is not a Cauchy sequence, then there exist a $\varepsilon>0$ and two sequences $\{n(k)\}$ and $\{m(k)\}$ of positive integers such that the following sequences tend to $\varepsilon^{+}$when $k \rightarrow \infty$ : 


$$
\begin{array}{ll}
d\left(x_{m(k)-j(k)}, x_{n(k)}\right), & d\left(x_{m(k)-j(k)+1}, x_{n(k)}\right), \\
d\left(x_{m(k)-j(k)}, x_{n(k)+1}\right), & d\left(x_{m(k)-j(k)+1}, x_{n(k)+1}\right),
\end{array}
$$

where $j(k) \in\{1,2, \ldots, p\}$ is chosen so that $n(k)-(m(k)-j(k)) \equiv 1(\bmod p)$, for each $k \in \mathbb{N}$.

Proof If $\left\{x_{n}\right\}$ is not a Cauchy sequence, then there exist $\varepsilon>0$ and sequences $\{n(k)\}$ and $\{m(k)\}$ of positive integers such that

$$
n(k)>m(k)>k, \quad d\left(x_{m(k)}, x_{n(k)-1}\right)<\varepsilon, \quad d\left(x_{m(k)}, x_{n(k)}\right) \geq \varepsilon
$$

for all positive integers $k$. Then using (2.2) and the triangle inequality, we get

$$
\varepsilon \leq d\left(x_{m(k)}, x_{n(k)}\right) \leq d\left(x_{m(k)}, x_{n(k)-1}\right)+d\left(x_{n(k)-1}, x_{n(k)}\right)<\varepsilon+d\left(x_{n(k)-1}, x_{n(k)}\right) .
$$

Passing to the limit as $k \rightarrow \infty$ in the above inequality and using (2.1), we obtain

$$
\lim _{k \rightarrow \infty} d\left(x_{m(k)}, x_{n(k)}\right)=\varepsilon^{+} .
$$

Note that, by the way the $j(k)$ were chosen, $x_{m(k)-j(k)}$ and $x_{n(k)}$ (for $k$ large enough, $m(k)>$ $j(k))$ lie in different adjacently labeled sets $A_{i}$ and $A_{i+1}$ for certain $i \in\{1,2, \ldots, p\}$. This will be used further in the proofs of Theorems 2.3 and 2.7.

Using the triangle inequality, we get

$$
\begin{aligned}
& \left|d\left(x_{m(k)-j(k)}, x_{n(k)}\right)-d\left(x_{m(k)}, x_{n(k)}\right)\right| \\
& \quad \leq d\left(x_{m(k)-j(k)}, x_{m(k)}\right) \\
& \quad \leq \sum_{l=0}^{j(k)-1} d\left(x_{m(k)-j(k)+l}, x_{m(k)-j(k)+l+1}\right) \rightarrow 0 \quad \text { as } k \rightarrow \infty
\end{aligned}
$$

(from (2.4)), which, by (2.5), implies that

$$
\lim _{k \rightarrow \infty} d\left(x_{m(k)-j(k)}, x_{n(k)}\right)=\varepsilon
$$

Using (2.1), we have

$$
\lim _{k \rightarrow \infty} d\left(x_{m(k)-j(k)+1}, x_{m(k)-j(k)}\right)=0 \quad \text { and } \quad \lim _{k \rightarrow \infty} d\left(x_{n(k)+1}, x_{n(k)}\right)=0 .
$$

Again using the triangle inequality, we get

$$
\left|d\left(x_{m(k)-j(k)}, x_{n(k)+1}\right)-d\left(x_{m(k)-j(k)}, x_{n(k)}\right)\right| \leq d\left(x_{n(k)+1}, x_{n(k)}\right) .
$$

Passing to the limit as $k \rightarrow \infty$ in the above inequality, and using (2.1) and (2.6), we get

$$
\lim _{k \rightarrow \infty} d\left(x_{m(k)-j(k)}, x_{n(k)+1}\right)=\varepsilon
$$


Similarly, we have

$$
\lim _{k \rightarrow \infty} d\left(x_{m(k)-j(k)+1}, x_{n(k)+1}\right)=\varepsilon .
$$

If $p=1$ we obtain the following known result.

Corollary 2.2 [8] Let $(X, d)$ be a metric space and let $\left\{x_{n}\right\}$ be a sequence in $X$ such that

$$
\lim _{n \rightarrow \infty} d\left(x_{n+1}, x_{n}\right)=0
$$

If $\left\{x_{n}\right\}$ is not a Cauchy sequence, then there exist an $\varepsilon>0$ and two sequences $\{n(k)\}$ and $\{m(k)\}$ of positive integers such that the following sequences tend to $\varepsilon^{+}$when $k \rightarrow \infty$ :

$$
\begin{aligned}
& d\left(x_{m(k)}, x_{n(k)}\right), \quad d\left(x_{m(k)}, x_{n(k)+1}\right), \quad d\left(x_{m(k)-1}, x_{n(k)}\right), \\
& d\left(x_{m(k)-1}, x_{n(k)+1}\right), \quad d\left(x_{m(k)+1}, x_{n(k)+1}\right), \quad d\left(x_{m(k)+1}, x_{n(k)}\right), \ldots .
\end{aligned}
$$

Now, we announce our first result (probably new because of using the new lemma, Lemma 2.1 with the comparison function). It generalizes several known results in the literature [9-12].

Theorem 2.3 Let $(X, d)$ be a complete metric space, $\varphi:[0, \infty) \rightarrow[0, \infty)$ a comparison function and $f: X \rightarrow X$. If

$$
d(f(x), f(y)) \leq \varphi(d(x, y))
$$

for all $x, y \in X$, then $f$ has a unique fixed point $z \in X$ and all sequences of Picard iterates defined by $f$ converge to $z$.

Proof If $x_{0}$ is an arbitrary point from $X$ we can consider a Picard sequence as follows:

$$
x_{n+1}=f\left(x_{n}\right), \quad n=0,1,2, \ldots
$$

If $x_{n+1}=x_{n}$ for some $n$, then $x_{n}$ is a fixed point of $f$. So, we will suppose that $x_{n+1} \neq x_{n}$ for all $n$.

Now, applying (2.7) putting $x=x_{n}$ and $y=x_{n-1}$, we obtain

$$
\begin{aligned}
d\left(x_{n+1}, x_{n}\right) & =d\left(f\left(x_{n}\right), f\left(x_{n-1}\right)\right) \leq \varphi\left(d\left(x_{n}, x_{n-1}\right)\right) \\
& \leq \varphi^{2}\left(d\left(x_{n-1}, x_{n-2}\right)\right) \leq \cdots \leq \varphi^{n}\left(d\left(x_{1}, x_{0}\right)\right) \rightarrow 0, \quad \text { as } n \rightarrow \infty .
\end{aligned}
$$

Since $d\left(x_{n+1}, x_{n}\right) \rightarrow 0$ we can prove that $\left\{x_{n}\right\}$ is a Cauchy sequence in the space $(X, d)$ by using Corollary 2.2. Indeed, suppose that $\left\{x_{n}\right\}$ is not a Cauchy sequence. Then Corollary 2.2 implies that there exist $\varepsilon>0$ and two sequences $n(k)$ and $m(k)$ of positive integers such that

$$
n(k)>m(k)>k, \quad d\left(x_{m(k)}, x_{n(k)-1}\right)<\varepsilon, \quad d\left(x_{m(k)}, x_{n(k)}\right) \geq \varepsilon .
$$


Now, using (2.7) with $x=x_{m(k)}$ and $y=x_{n(k)-1}$, we get

$$
d\left(x_{m(k)+1}, x_{n(k)}\right) \leq \varphi\left(d\left(x_{m(k)}, x_{n(k)-1}\right)\right) \leq \varphi(\varepsilon)<\varepsilon
$$

(for each comparison function $\varphi: \varphi(t)<t$ for all $t>0$ ).

Taking the limit in (2.9) as $k \rightarrow \infty$ and using Corollary 2.2, we get

$$
\varepsilon=\lim _{k \rightarrow \infty} d\left(x_{m(k)+1}, x_{n(k)}\right) \leq \varphi(\varepsilon)<\varepsilon,
$$

which is a contradiction. Hence, $\left\{x_{n}\right\}$ is a Cauchy sequence. Therefore, there exists $z \in X$ such that $x_{n} \rightarrow z$. Since, according (2.7) $f$ is a continuous, we see that $f(z)=z$ is a fixed point of $f$. Uniqueness follows easily from (2.7). Theorem 2.3 is proved.

Remark 2.4 It is clear that Theorem 2.3 holds if $\varphi$ is also (c)-comparison function. It this case, it follows that the main result from [6]-Theorem 1.8 above is a cyclic-type extension of Theorem 2.3, where $\varphi$ is a (c)-comparison function. Also, if $\varphi$ is a (c)-comparison function in our Theorem 2.3 we have the same error estimates (1.4)-(1.6) as in [6]-Theorem 1.8.

Now, we shall prove that Theorem 1.8 and Theorem 2.3 with a (c)-comparison function $\varphi$ are equivalent, that is, Theorem 1.8 holds if and only if Theorem 2.3 holds.

Theorem 2.5 Theorem 1.8 and Theorem 2.3 with (c)-comparison function are equivalent.

Proof First of all, Theorem 1.8 implies Theorem 2.3. Indeed, putting in Theorem $1.8 A_{i}=X$ for all $i=1,2, \ldots, m$ we obtain result.

Conversely, we shall show that Theorem 2.3 implies Theorem 1.8. If $x_{0} \in \bigcup_{i=1}^{m} A_{i}$ then Picard's sequence $\left\{f^{n} x_{0}\right\}$ is obviously a Cauchy sequence (the proof is as in [6]) and converges to some $z \in \bigcup_{i=1}^{m} A_{i}$. Also, it is clear that infinitely many terms of $\left\{f^{n} x_{0}\right\}$ lie in each $A_{i}$. From this it follows that $\bigcap_{i=1}^{m} A_{i} \neq \emptyset$, because the $z$ lie in each $A_{i}$. Further, since (according to (1.1)) $f: \bigcap_{i=1}^{m} A_{i} \rightarrow \bigcap_{i=1}^{m} A_{i}$, the restriction $\left.f\right|_{\bigcap_{i=1}^{m} A_{\mathbf{i}}}$ of $f$ on $\bigcap_{i=1}^{m} A_{i}$ satisfies (2.7) and $\left(\bigcap_{i=1}^{m} A_{i}, d\right)$ is a complete metric space, Theorem 2.3, where $\varphi$ is a (c)-comparison function, implies that the mapping $f$ has a unique fixed point in $\bigcap_{i=1}^{m} A_{i}$, that is, Theorem 1.8 holds. Theorem 2.5 is proved.

Remark 2.6 From (II) of Definition 1.1 it follows that $f: \bigcap_{i=1}^{m} A_{i} \rightarrow \bigcap_{i=1}^{m} A_{i}$ in the case that $\bigcap_{i=1}^{m} A_{i} \neq \emptyset$. Further, if Picard's sequence $\left\{f^{n} x\right\}$, where $f: \bigcup_{i=1}^{m} A_{i} \rightarrow \bigcup_{i=1}^{m} A_{i}, x \in \bigcup_{i=1}^{m} A_{i}$ is Cauchy, then $\bigcap_{i=1}^{m} A_{i} \neq \emptyset$. Also, if such $f$ has a fixed point, say $z$, then by (II) of Definition 1.1 it follows that $z \in \bigcap_{i=1}^{m} A_{i}$, that is,

$$
\operatorname{Fix}(f) \neq \emptyset \Rightarrow \bigcap_{i=1}^{m} A_{i} \neq \emptyset
$$

This follows directly from the conditions $f\left(A_{i}\right) \subseteq A_{i+1}, i=1,2, \ldots, m$.

If a certain (non-cyclic) fixed point result is known, in order to obtain the respective cyclic-type fixed point result, it is enough to prove that the respective cyclic contractive 
condition implies that $\bigcap_{i=1}^{m} A_{i} \neq \emptyset$. Indeed, in this case $\left(\bigcap_{i=1}^{m} A_{i}, d\right)$ is a complete metric space and the restriction of $f$ to $\bigcap_{i=1}^{m} A_{i}$ satisfies the given standard condition.

By using Lemma 2.1 we prove the following result.

Theorem 2.7 Theorem 1.12 and Theorem 1.13 are equivalent.

Proof It is clear that Theorem 1.13 is true, if Theorem 1.12 is. Conversely, we will prove that Theorem 1.13 implies Theorem 1.12. Indeed, if $x_{0} \in \bigcup_{i=1}^{m} A_{i}$, then for the Picard sequence $x_{n}=f\left(x_{n-1}\right), x_{n} \neq x_{n-1}, n=1,2, \ldots$ we have as in [7] that $d\left(x_{n+1}, x_{n}\right) \downarrow 0$.

Now, putting in (II), Definition 1.1, $x=x_{m(k)-j(k)}, y=x_{n(k)}$ we obtain

$$
\begin{aligned}
\mu\left(d\left(x_{m(k)-j(k)+1}, x_{n(k)+1}\right)\right) \leq & \mu\left(\frac{1}{2}\left(d\left(x_{m(k)-j(k)}, x_{n(k)+1}\right)+d\left(x_{m(k)-j(k)+1}, x_{n(k)}\right)\right)\right) \\
& -\psi\left(d\left(x_{m(k)-j(k)}, x_{n(k)+1}\right), d\left(x_{m(k)-j(k)+1}, x_{n(k)}\right)\right) .
\end{aligned}
$$

Taking the limit in (2.11) as $k \rightarrow \infty$, we get by Lemma 2.1

$$
\mu(\varepsilon) \leq \mu\left(\frac{1}{2}(\varepsilon+\varepsilon)\right)-\psi(\varepsilon, \varepsilon)=\mu(\varepsilon)-\psi(\varepsilon, \varepsilon)
$$

that is, $\psi(\varepsilon, \varepsilon)=0$. According to the property of the function $\psi$, it follows that $\varepsilon=0$. A contradiction. Hence, the Picard sequence $x_{n}=f\left(x_{n-1}\right)$ is Cauchy. Further, according to Remark 2.6 we see that Theorem 1.13 implies Theorem 1.12. The proof of Theorem 2.7 is completed.

\section{An open question:}

Prove or disprove the following:

- Let $\left\{A_{i}\right\}_{i=1}^{m}$ be non-empty closed subsets of a complete metric space, and suppose $f: \bigcup_{i=1}^{m} A_{i} \rightarrow \bigcup_{i=1}^{m} A_{i}$ satisfies the following conditions (where $A_{m+1}=A_{1}$ ):

(1) $f\left(A_{i}\right) \subseteq A_{i+1}$ for $1 \leq i \leq m$ and $f\left(A_{m}\right) \subseteq A_{1}$;

(2) there exists a comparison function $\varphi:[0, \infty) \rightarrow[0, \infty)$ such that

$$
d(f(x), f(y)) \leq \varphi(d(x, y))
$$

for all $x \in A_{i}, y \in A_{i+1}, 1 \leq i \leq m$.

Then $f$ has a unique fixed point $x^{*} \in \bigcap_{i=1}^{m} A_{i}$ and Picard iteration $\left\{x_{n}\right\}_{n \geq 1}$ given by $x_{n}=f\left(x_{n-1}\right)$ converge to $x^{*}$ for any starting point $x_{0} \in \bigcup_{i=1}^{m} A_{i}$.

\section{Competing interests}

The author declares that he has no competing interests.

\section{Acknowledgements}

The author is grateful to the referees of this paper, whose suggestions helped to improve the text in many places.

Received: 29 June 2015 Accepted: 8 October 2015 Published online: 21 October 2015

\section{References}

1. Banach, S: Sur les opérations dans les ensembles abstraits et leur applications aux équations intégrales. Fundam. Math. 3, 133-181 (1922)

2. Kirk, WA, Srinivasan, PS, Veeramani, P: Fixed points for mappings satisfying cyclical contractive conditions. Fixed Point Theory 4(1), $79-89$ (2003) 
3. Nashine, HK: Cyclic generalized $\psi$-weakly contractive mappings and fixed point results with applications to integral equations. Nonlinear Anal. 75, 6160-6169 (2012)

4. Radenović, S: Some remarks on mappings satisfying cyclical contractive conditions. Afr. Math. (2015) doi:10.1007/s13370-015-0339-2

5. Radenović, S: A note on fixed point theory for cyclic weaker Meir-Keeler function in complete metric spaces. Int. J. Anal. Appl. 7(1), 16-21 (2015)

6. Pacurar, M, Rus, IA: Fixed point theory for cyclic $\varphi$-contractions. Nonlinear Anal. 72, 1181-1187 (2010)

7. Chandok, S, Postolache, M: Fixed point theorem for weakly Chatterjea-type cyclic contractions. Fixed Point Theory Appl. 2013, 28 (2013)

8. Radenović, S, Kadelburg, Z, Jandrlić, D, Jandrlić, A: Some results on weak contraction maps. Bull. Iran. Math. Soc. 38(3), 625-645 (2012)

9. Browder, FE: On the convergence of successive approximations for nonlinear functional equations. Indag. Math. 30 27-35 (1968)

10. Boyd, DW, Wong, JS: On nonlinear contractions. Proc. Am. Math. Soc. 20, $458-464$ (1969)

11. Matkowski, J: Integrable solutions of functional equations. Diss. Math. 127 (1975) 68 pp.

12. Rus, IA: Generalized $\varphi$-contractions. Mathematica 24, 175-178 (1982)

\section{Submit your manuscript to a SpringerOpen ${ }^{\mathcal{O}}$ journal and benefit from:}

- Convenient online submission

Rigorous peer review

- Immediate publication on acceptance

- Open access: articles freely available online

- High visibility within the field

- Retaining the copyright to your article 